\title{
Reconstrucción osteobiográfica en odontología forense
}

\author{
Osteo biographical reconstruction in forensic dentistry
}

\section{Resumen}

En las Ciencias Forenses la estimación de la Reconstrucción Osteobiográfica de un sujeto forma parte importante del complejo proceso de identificación humana. En la Reconstrucción Osteobiográfica toma en cuenta los métodos para la estimación de la edad, el sexo, los ancestros y la estatura basados principalmente en el estudio del macizo cráneofacial, su morfología, dimensiones métricas apoyan a las ciencias forenses con una importante batería de datos que contribuyen a la individualización de personas desaparecidas. En este sentido, la carta dental que se aplica regularmente en la identificación es una mínima parte de toda la información que podemos obtener del sistema dental. Aquí podemos decir, al igual que para los huesos, el macizo cráneofacial y dentro de ello los dientes hablan y cuentan la historia de la persona desaparecida que en vida formó parte de un medio ambiente, un grupo social determinado y de una individualidad que lo hace único e irrepetible.

Palabras clave: odontología forense, medidas antropométricas, estimación de la estatura, valoración de la edad dental,

\section{Abstract}

In forensic science estimating osteo biographical reconstruction of a subject is an important part of the complex process of human identification. Osteo biographical reconstruction takes into account the methods for estimating age, sex, ancestry and stature based mainly on the study of craniofacial skull, morphology, metric dimensions, which support forensic science with an important battery data contributing to the identification of missing persons. In this sense, the dental chart which is regularly applied in identification is a fraction of all the information we can get the dental system. Here we can say, as for the bones, the cranium and teeth speak and tell the story of the missing person that was part of a living environment, a particular social group and an individuality that makes unique.

Keywords: forensic dentistry, anthropometric measures, stature estimation, assessment of dental age

\section{Introducción}

La aplicación de los conocimientos de Odontología Forense ha demostrado ser de gran utilidad en la identificación humana, pues se basan principalmente en aspectos fisiológicos y en las variaciones adquiridas del macizo craneofacial como reflejo de la actividad socioeconómica, cultural, biológica del hombre, lo que permite la elaboración de técnicas especiales para estos fines, que unidas a las que aportan otras disciplinas, logran la identificación de los restos. Esta característica permite que el macizo craneofacial y dentro de ello las piezas dentarias, sean el elemento más importante a tener en cuenta durante el proceso de identificación humana, sobre todo cuando las estructuras blandas del organismo no sean factibles de ser usadas en dicho proceso.

Ante la presencia de cadáveres no identificados, pudiéndose encontrar estos en diferentes formas: carbonizados, mutilados, en avanzado estado de putrefacción, momificados o en estado de esqueletización y cuando el método dactiloscópico, odontograma de identificación, el ADN no sean aplicables, se utilizarán los métodos odontoantropológicos, las cuales ayudarán y cobraran importancia en el proceso de identificación humana, analizándose sus restos óseos y dentales con el fin de reconstruir la osteobiografía (edad, sexo, ancestros, estatura), aportando datos que contribuirán a la individualización de las personas que han perdido su identidad civil.

\section{Revisión de la literatura}

Metodología de la Identificación $\mathrm{Hu}-$ mana

La normativa legal en los casos de muerte violenta reconocía la importancia de identificar al fallecido apelando a la "identificación de visu" o reconocimiento visual del cadáver por testigos confiables. Sin embargo, el reconocimiento visual ya sea que la persona observe el cuerpo directamente o que examine fotografías, tiene un alto riesgo de error que se explica por el estado emocional alterado de quien enfrenta tal situación, por los cambios que haya podido presentar el cadáver y por la calidad de las fotografías. ${ }^{1}$ En
Daniel Guillermo Suárez Ponce ${ }^{1}$
1. Departamento Académico Médico Quirúrgico, Facultad de Odontología, Universidad Nacional Mayor de San Marcos, Perú.

\section{Correspondencia}

Mg. Daniel Guillermo Suárez Ponce

Facultad de Odontología. Universidad Nacional Mayor de San Marcos. Av. Germán Amézaga s/n. Lima 1. Perú

Correo electrónico:

daniel06suarez@hotmail.com
Fecha de recepción: 30-11-12

Fecha de aceptación: 19-06-14 los procedimientos de identificación de personas desaparecidas sin documentos de identidad, al igual que cuando se estudian cadáveres y no se cuenta con huellas dactilares ni fotografías de los cadáveres, se analizan sus restos óseos y dentales a fin de reconstruir la osteobiografía brindando una importante información sobre la edad, sexo, ancestros, lateralidad, hábitos alimenticios, deficiencias durante el desarrollo de los niños, aspectos genéticos e histomorfométricos; su morfología, dimensiones métricas, el patrón de desgaste, los tratamientos dentales y la cavidad pulpar que es una cámara de conservación de material genético, apoyan las ciencias forenses con una importantísima batería de datos que contribuyen a la individualización de personas desaparecidas.

\section{Metodología de la estimación del sexo}

La estimación del sexo en los restos óseos es muy importante para llegar a la identificación del sujeto. Es importante señalar que las características y rasgos de cada hueso, son relativamente determi- 
nantes para la estimación del sexo, por lo que siempre se debe tener en cuenta la suma de todas las características y no una sola aislada en el contexto. La determinación del sexo es más segura en adultos que en subadultos, siendo las diferencias morfológicas consecuencia de la influencia de hormonas como el estrógeno o la testosterona, que fuertemente afectan a los huesos en la pubertad en adelante. ${ }^{2}$

El cráneo no es la región del esqueleto cuyo sexo resulta más fácil de determinar, sobre todo si se encuentra roto o fragmentado. ${ }^{3}$ Los tipos difieren por el rango de la variabilidad de los criterios específicos cresta nucal, proceso mastoide, borde supraorbital, arco supraorbital y prominencia del mentón en el contexto del sexamiento, publicado por Buikstra \& Ubelaker (1994) en los estándares para la recolección de datos fundamentado en restos óseos. ${ }^{4}$

El dimorfismo sexual en los dientes es muy variable, normalmente los dientes en el sexo femenino suelen ser más pequeños, sobre todo el diámetro mesiodistal de los molares permanentes, pero a veces es muy difícil distinguirlos por este único rasgo. La primera característica que se tendría que considerar sería la dimensión o medidas dentales (odontometría). En un estudio realizado por Toribio Suárez y colaboradores se señaló que existen también diferencias morfológicas entre patrones raciales. $^{5}$

En estudios odontométricos, como el realizado por Gómez Hernández ${ }^{6}$ el 2002, encontró que las medidas de los caninos superiores tienen un alto grado de dimorfismo sexual, especialmente la altura radicular y el diámetro vestíbulo-palatino; también confirmó las diferencias morfológicas entre poblaciones. Es muy difícil saber el sexo, por un solo diente de la persona a quien corresponde; en general las mujeres los tienen mucho más pequeños que los hombres, más suaves y claros. ${ }^{7}$

Loth y Henneberg ${ }^{8}$ (1996), encontraron que los individuos adultos masculinos exhibían una angulación distintiva en el bordeposterior de la rama vertical. El estudio cefalométrico lateral y frontal ayudan en la determinación del sexo basado en el estudio del análisis de las funciones discriminantes en radiografías cefalométricas, entre los principales parámetros usados son el bizigomático, con la altura de la rama y anchura de la cara contribuyen en la predicción del dimorfismo sexual.
Metodología de la estimación de la edad

El desarrollo dental posee el potencial para la estimación de la edad durante toda la niñez. Normalmente, los resultados son más precisos para niños menores a causa de la mineralización determinada y menor influencia ambiental (como malnutrición aguda) que pueden además provocar caries o pérdida de piezas dentales. Como se ha mencionado anteriormente, el desarrollo dental es controlado por factores genéticos y sufre por ello poca influencia ambiental (Smith 1991, Ubelaker 1999). ${ }^{9,10}$

Aunque el proceso es continuo, es conveniente dividirlo en distintas etapas, basadas en la cantidad de la calcificación. El esquema más común es el de Moorrees y colaboradores para dentadura decidual y permanente con base en dientes de una raíz (incisivos, caninos, premolares) y de múltiples raíces (molares). ${ }^{11}$ Otro esquema fue presentado por Demirjian(1978)(23) y Demirjian y otros $(1973,1976)^{12,13}$, que toma en cuenta una escala del desarrollo dental descrito desde A hasta $\mathrm{H}$.

Schour \& Massler publicaron su estudio inicial con un diagrama sobre el desarrollo dental, vinculando tanto la formación como la erupción de las piezas dentales. ${ }^{14,15} \mathrm{El}$ esquema elaborado por Ubelaker (1978, 1999) es el más recomendable para la utilización en casos de población amerindia, siendo su estudio más reconocido por la comunidad científica que parece ser la mejor metodología para este grupo étnico. ${ }^{10,16}$

En individuos adultos el desgaste dental en restos óseos sirve para estimar la edad, desde la escala de cinco grados propuesta por Broca (Alexeev, Debetz, 1964) a finales del siglo pasado, diferentes autores han tratado de medir el desgaste, correlacionándolo con patrones de cambio (Murphy, 1959; Brothwell, 1989), la función y variabilidad cultural (Molnar, 1971), técnicas de medición (Guerasimov, 1955; Zoubov, 1968; Alexeev y Debetz, 1964; Scott, 1979; Lovejoy, 1985), la enumeración de anillos en el cemento (Naylor et al., 1985) y el gradiente del grosor del esmalte (Macho, Berner, 1993). ${ }^{17}$

En 1950, Gustafson ${ }^{18}$ observó que la dentina se hacía transparente con la edad, proponiendo un procedimiento bastante complejo que ha sido discutido y modificado desde su propuesta (Johanson, 1971; Maples, 1978; Kilian \& Vlcek, 1989, Lamendin, 1988, Prince \& Ubelaker, 2002; entre otros). ${ }^{17}$ La propuesta de Lamendin (1988) es la medición de regresión total de la periodontitis, la transparencia y la altura de la raíz. ${ }^{19}$ La técnica de Lamendin (1988) es la modificación simplificada del método original de G. Gustafson (1947). Posteriormente, D. Prince \& D. Ubelaker realizaron pruebas de verificación expresando la edad como una función de dos factores: la translucidez de la raíz del diente y periodontosis (la regresión del gingival).

\section{Metodología de la estimación de la Estatura}

Desde hace tiempo, la reconstrucción de la estatura que toma como base el material óseo era de interés de antropólogos. La estatura o talla de pie se define como la altura comprendida entre el vértex, el punto más elevado de la cabeza hasta el suelo, orientando la cabeza del individuo en el plano de Frankfort. Se suele medir mediante el antropómetro y se expresa la medición en centímetros ${ }^{20}$

Trotter \& Gleser ${ }^{21,22}(1952,1958)$ han computado fórmulas de ecuación tentativas para estimar la estatura base en longitudes de las extremidades superiores e inferiores. Genovés ${ }^{23}$ (1967) estudió la variación de la estatura en una muestra de cadáveres mejicanos estableciendo fórmulas de correlación entre la longitud de los huesos largos y la estatura para indígenas centroamericanos.

La estatura se puede estimar a través de las dimensiones de cada diente son proporcionales al conjunto que forma la arcada dentaria, cabeza y el sujeto total. Carrea confeccionó una tabla y unas fórmulas que permiten la reconstrucción de las principales medidas antropométricas, y especialmente de la talla del individuo en casos de despedazamiento o descuartizamiento criminal. En la estimación de la estatura las medidas de la región cefálica presentan fuerte correlación y mejor predicción de la estatura en referencia a las medidas de la región facial. Siendo factible el establecimiento de fórmulas de regresión.

Metodología de la Estimación de los Ancestros $^{24}$

En investigaciones forenses se trabaja con poblaciones contemporáneas en las cuales los grupos raciales suelen hallarse mezclados en un solo individuo, 
por lo que se trata de establecer cuál de los tres grandes grupos raciales (caucasoide, negroide y mongoloide) predomina, evaluando características métricas y morfológicas en el cráneo que es la zona del esqueleto más adecuada para valorar características raciales.

Los métodos no métricos, son los más utilizados, ya que no requieren un equipo específico y las observaciones se hacen rápidamente; entre los indicadores más utilizados se encuentran: la distancia intraorbital, las características de huesos nasales, apertura nasal, el prognatismo alveolar, la proyección de los malares y la morfología palatina de incisivos (presencia o ausencia de forma de pala).

Con la excepción de los dientes, especialmente los incisivos superiores, la mayoría de las características solamente permiten una investigación en el marco del patrón ancestral en esqueletos adultos. Para esto se analizan criterios morfológicos, en primer lugar del cráneo por la antroposcopía - la inspección visual - y morfométricos de los restos esqueletizados.

Entre los diferentes grupos de ascendencia existen varios rasgos que se manifiestan en los huesos, especialmente en el cráneo. El problema con estas características es la subjetividad en el marco de la observación, por lo cual se necesita estudiarlas en categorías discretas o no-continuas y no se miden objetivamente, o declararlas en gradaciones. El rasgo craneal que muestra las mayores diferencias entre los grupos étnicos es la apertura nasal, especialmente la raíz, el puente, la espina, el borde inferior y la forma de la apertura nasal.

\section{Conclusiones}

La Reconstrucción Osteobiográfica tiene un papel muy importante en el proceso de identificación humana determinando principalmente la edad, sexo, ancestros y la estatura.

Los procedimientos de la Reconstrucción Osteobiográfica se emplearan en la identificación humana cuando los métodos de identificación fehacientes (dactiloscopia, métodos odontológicos y el ADN) no sean aplicables

\section{Referencias bibliográficas}

1. Jimenez G. Manual para la Identificación de cadáveres. Instituto Nacional de Medicina Legal y
Ciencias Forenses. Colombia. 2005;9-12.

2. Krogman WM \& MY Iscan. The human skeleton in forensic medicine. Springfield, Illinois. CC. Thomas Pub.1986

3. 3. Brothwell DR. Digging up bones. The excavation, treatment and study of human skeletal remains. 3rd ed. Ithaca, New York: Cornell University Press. (1987)

4. 4. Buikstra, J. \& Ubelaker, D. Standards, For Data Collection from Human Skeletal Remains. Arkansas Archeological survey research series $\mathrm{N}^{\circ}$ 44. (1994)

5. Suárez T, Quezada R, Calle R. Identificación del sexo y el grupo racial por mediciones dentarias. Estudios de antropología biológica. 1995; No 05.

6. F Gómez Hernández L. Determinación del sexo a partir de odontometría de canino superior izquierdo en población mestiza colombiana de sala de necropsias [tesis de posgrado en Odontología Legal y Forense]. Bogotá: Pontificia Universidad Javeriana; 2002.

7. igón ME, Garino RR. Anatomía Odontológica funcional y aplicada. 2a Ed. Buenos Aires. El ateneo. 1988 .

8. Loth SR and Henneber M. Mandibular Ramus Flexure: A New Morphologic Indicator of Sexual Dimorphism in the Human Skeleton. Am J Phys Anthropol. 1996;99:473 - 485.

9. Smith, B.H. Standars of Humam tooth formation and dental age assessment. En Kelly, M. A. \& Larsen, CS, editores. Advances in dental anthropology. New York: Wiley-Liss;1991. Pp.143 -168 .

10. Ubelaker DH. Human Skeletal Remains. Excavation, Analysis, Interpretation. TaraxacumWashington, Smiths. Inst. Manuals on Archaeology; 1999.

11. Moorrees CF, Fanning EA \& Hunt EE. Age variation of Formation Stages for ten permanent teeth. Journal of Dental Research. 1963;42:1490-1502.
12. Demirjian A, Goldstein H. New systems for dental maturity based on seven and four teeth. Ann. Hum. Biol. 1976;3(5):411-421.

13. Demirjian A, Goldstein H, Tanner JM. A new system of dental age assessment. Ann. Hum. Biol. 1973;45:211-227.

14. Schour I, Massler M. The development of the human dentition. Journal of the American Dental Association. 1941;20:379-427.

15. Schour I, Massler M. Studies in tooth development. The growth pattern of human teeth. Journal of the American Dental Association. 1940;27:1918-1931.

16. Ubelaker DH. Human skeletal remains. Taraxacum-Washington, Smiths. Inst. Manuals on Archaeology; 1978.

17. Rodríguez JV. Dientes y Diversidad Humana. Avances de la Antropología Dental. Colombia: Editora Guadalupe Ltda.; 2003.

18. Gustafson G. Age determination on teeth. Journal of the American dental Association. 1950;41:4554.

19. Lamendin H. Age determination with the "simplified" Gustafson Method. Chir Dent Fr. 1988;58(427):43-47.

20. 20. Byers SN (2001) Introduction in forensic anthropology. A textbook. Allyn and Bacon, Boston, Sydney, Tokyo.

21. 21. Trotter M \& GC Gleser. The effects of ageing on stature. American Journal of Physical Anthropology. 1951;9:311-324.

22. Trotter M \& GC Gleser. A reevaluation of estimation of stature based an measurements of stature taken during life and of long bones after death. American Journal of Physical Anthropology. 1958;16:79-123.

23. Genovés S. Proportionality of long bones and their relation to stature among Mesoamericans. American Journal of Physical Anthropology. 1967;26:67-77.

24. Krenzer U. Compendio de métodos antropológico forenses para la reconstrucción del perfil osteo-biológico. Guatemala; 2006. 